\title{
Higher Bruhat Orders in Type B
}

\author{
Seth Shelley-Abrahamson \\ Department of Mathematics \\ Massachusetts Institute of Technology \\ Cambridge, MA, USA \\ sethsa@mit.edu
}

\author{
Suhas Vijaykumar \\ Department of Mathematics \\ Massachusetts Institute of Technology \\ Cambridge, MA, USA \\ suhasv@mit.edu
}

Submitted: Oct 7, 2015; Accepted: Jun 30, 2016; Published: Jul 22, 2016

Mathematics Subject Classifications: 06A07, 20F55

\begin{abstract}
Motivated by the geometry of hyperplane arrangements, Manin and Schechtman defined for each integer $n \geqslant 1$ a hierarchy of finite partially ordered sets $B\left(I_{n}, k\right)$, indexed by positive integers $k$, called the higher Bruhat orders. The poset $B\left(I_{n}, 1\right)$ is naturally identified with the weak left Bruhat order on the symmetric group $S_{n}$, each $B\left(I_{n}, k\right)$ has a unique maximal and a unique minimal element, and the poset $B\left(I_{n}, k+1\right)$ can be constructed from the set of maximal chains in $B\left(I_{n}, k\right)$. Ben Elias has demonstrated a striking connection between the posets $B\left(I_{n}, k\right)$ for $k=2$ and the diagrammatics of Bott-Samelson bimodules in type A, providing significant motivation for the development of an analogous theory of higher Bruhat orders in other Cartan-Killing types, particularly for $k=2$. In this paper we present a partial generalization to type $\mathrm{B}$, completed up to $k=2$, prove a direct analogue of the main theorem of Manin and Schechtman, and relate our construction to the weak Bruhat order and reduced expression graph for Weyl group $B_{n}$.
\end{abstract}

Keywords: Coxeter theory, poset, Bruhat order

\section{Introduction}

In their 1989 paper [4], Manin and Schechtman introduced for each positive integer $n$ a family of ranked partially ordered sets $B(n, k)$, the higher Bruhat orders, indexed by positive integers $k$. The motivation for this purely combinatorial construction came from the geometry of hyperplane arrangements, and the geometric picture was used to study these posets by Felsner-Weil [2], Kapranov-Voevodsky [3], Ziegler [6], and others. More recently, Elias [1] has demonstrated a striking connection between the higher Bruhat orders $B(n, k)$ for $k=2$ and the diagrammatrics of Bott-Samelson bimodules in type $A$, giving significant motivation for the development of an analogous theory of higher Bruhat orders in other Cartan-Killing types, particularly for $k=2$. 
In this paper, we will introduce such a partial generalization of the Manin-Schechtman construction in type $B$. In particular, a generalization to type $B$, in analogy with the construction in type $A$, should specify a family of sets $C_{B}\left(J_{n}, k\right)$ indexed by positive integers $n$ and $k$ along with a packet operation $P_{B}$ that associates to an element $K \in$ $C_{B}\left(J_{n}, k+1\right)$ a packet $P_{B}(K) \subset C_{B}\left(J_{n}, k\right)$. There should be a specified standard order on packets, leading to a notion of admissible orderings $A_{B}\left(J_{n}, k\right)$ of $C_{B}\left(J_{n}, k\right)$ as those total orderings of $C_{B}\left(J_{n}, k\right)$ whose restrictions to any packet extends either the standard order or its opposite on that packet. There should be an equivalence relation $\sim$ on $A_{B}\left(J_{n}, k\right)$ analogous to the equivalence relation defined by Manin-Schechtman in type $A$, and for each packet $K$ a packet flip operation $p_{K}(\bullet)$ defined on appropriate elements of $A_{B}\left(J_{n}, k\right)$ and descending to the quotient $B_{B}\left(J_{n}, k\right):=A_{B}\left(J_{n}, k\right) / \sim$. There should be a notion of inversion set for elements of $A_{B}\left(J_{n}, k\right)$, defined in terms of the standard order on packets, and this notion should respect the relation $\sim$ and therefore induce a notion of inversion set for elements of $B_{B}\left(J_{n}, k\right)$. This notion of inversion set, along with the packet flip operation, should endow $B_{B}\left(J_{n}, k\right)$ with the structure of a poset ranked by the cardinality of the inversion set, in a manner analogous to the original construction in type $A$. Finally, and most importantly, this data should be coherent as $k$ varies, in a manner analogous to the main theorem of Manin-Schechtman in type $A$. In particular, each poset $B_{B}\left(J_{n}, k\right)$ should have a unique maximal element and a unique minimal element, $A_{B}\left(J_{n}, k+1\right)$ should be naturally identified with the set of maximal chains in $B_{B}\left(J_{n}, k\right)$, and any element of $B_{B}\left(J_{n}, k\right)$ should be determined by its inversion set.

Such a construction should be considered a "type $B$ " higher Bruhat order if the connections between the Manin-Schechtman higher Bruhat orders and the combinatorics of the type $A$ Weyl groups hold instead for the type $B$ Weyl groups. In section 3 we make explicit the connection at the levels $k=1,2$ between the higher Bruhat orders and the combinatorics of type $A$ Weyl groups. In particular, we explain that the ranked poset $B\left(I_{n}, 1\right)$ of Manin-Schechtman is naturally identified with the symmetric group $S_{n}$ on $n$ letters with its natural weak left Bruhat order, ranked by the number of inversions; that the set $C\left(I_{n}, 2\right)$ is naturally identified with a set of positive roots in the root system of $S_{n}$ in a manner compatible with inversion sets; that the set of maximal chains in $B\left(I_{n}, 1\right)$, and hence the set $A\left(I_{n}, 2\right)$ by the main theorem of Manin-Schechtman, is naturally identified with the set of reduced expressions for the longest element of $S_{n}$ as a Coxeter group; and that the equivalence relation $\sim$ on $A\left(I_{n}, 2\right)$ is generated by $s t=t s$ braid relations and that the packet flip operation correspond to sts $=t s t$ braid relations. All of these notions have natural analogues for type $B$ Weyl groups which serve as the motivation for the definitions we give in section 4 at the levels $k=1,2$, and we see in Theorem 20 and Corollary 21 that the construction we give there is compatible with the combinatorics of the type $B$ Weyl groups in the same way. In section 4 , we provide sufficient definitions at the levels $k=3,4$ to formulate and prove the analogue of the main theorem of ManinSchechtman at the levels $k=1,2$, which is achieved in Proposition 7 and Theorems 9 and 15.

Motivated by the connection with categorification in [1] mentioned above, we have focused our efforts here on developing the theory at the levels $k=1,2$ sufficient for that 
purpose. However, it should be be very interesting to complete this generalization and the proof of the analogue of the main theorem of Manin-Schechtman for $k>2$ and to address in this context matters explored previously for the type $A$ higher Bruhat orders. For instance, following the work of Felsner-Weil [2], one may attempt to identify the partial order on the set $B_{B}\left(J_{n}, 2\right)$ introduced here with the partial order defined by inversion set containment, and similarly one may attempt to conduct a geometric study of these higher orders following, for instance, Ziegler's work [6] in type $A$. We comment that the reduced expression graphs in type $B$ have already received some attention, for example in the work of Reiner and Roichman [5]. Finally, it would be very interesting to determine the extent to which a theory of higher Bruhat orders can, or cannot, be developed for Weyl groups of type $D$ or of exceptional type.

\section{Higher Bruhat orders in type $A$ (cf. [4])}

In this section, we recall the construction of and main theorem for the original ManinSchechtman higher Bruhat orders.

Let $I_{n}:=\{1, \ldots, n\}$ be totally ordered in the usual way, and let $C\left(I_{n}, k\right)$ denote the set of $k$-element subsets of $I_{n}$. Then $C\left(I_{n}, k\right)$ is totally ordered by the lexicographic ordering, denoted $\rho_{\text {min }}$. We refer to the reverse total ordering as the anti-lexicographic ordering, denoted $\rho_{\max }$.

For each $K \in C\left(I_{n}, k+1\right)$, let $P(K):=\left\{S \in C\left(I_{n}, k\right): S \subset K\right\}$ be the set of all $k$ element subsets of $K$. We refer to $P(K)$ as the packet of $K$ and to any subset of $C\left(I_{n}, k\right)$ of the form $P(K)$ for some $K \in C\left(I_{n}, k+1\right)$ as a $k$-packet. Call a total ordering of $C\left(I_{n}, k\right)$ admissible if its restriction to each $k$-packet is either lexicographic or anti-lexicographic. Let $A\left(I_{n}, k\right)$ denote the set of admissible total orderings of $C\left(I_{n}, k\right)$. Clearly, if $\rho$ is an admissible ordering so is its reverse ordering $\rho^{t}$, and both the lexicographic ordering $\rho_{\min }$ the anti-lexicographic ordering $\rho_{\max }=\rho_{\min }^{t}$ are admissible. Note that any total ordering of $C\left(I_{n}, 1\right)=I_{n}$ is admissible, as the only total orderings on a 1-packet are the lexicographic and anti-lexicographic orderings, so the admissibility criterion is vacuous for $k=1$ and $A\left(I_{n}, 1\right)$ is the set of total orderings of $I_{n}$.

For a set $S$, let $2^{S}$ denote the set of subsets of $S$, and for a total ordering $\rho$ on $S$ and a subset $T \subset S$ let $\left.\rho\right|_{T}$ denote the restriction of $\rho$ to $T$. Let the function Inv $: A\left(I_{n}, k\right) \rightarrow$ $2^{C\left(I_{n}, k+1\right)}$ be defined by

$$
\operatorname{Inv}(\rho):=\left\{K \in C\left(I_{n}, k+1\right):\left.\rho\right|_{P(K)}=\left.\rho_{\max }\right|_{P(K)}\right\} .
$$

For example, $\operatorname{Inv}\left(\rho_{\min }\right)=\varnothing$ and $\operatorname{Inv}\left(\rho_{\max }\right)=C\left(I_{n}, k+1\right)$. For $k=1$ this gives the usual notion of the inversion set of a permutation of $I_{n}$.

Let the function $N: A\left(I_{n}, k\right) \rightarrow 2^{C\left(I_{n}, k+1\right)}$ be defined by

$$
N(\rho):=\left\{K \in C\left(I_{n}, k+1\right): P(K) \text { forms a chain in } \rho\right\} .
$$

For $\rho \in A\left(I_{n}, k\right)$ and $K \in N(\rho)$, the ordering $p_{K}(\rho)$ obtained by reversing the ordering of the chain $P(K)$ in $\rho$ is also admissible, because any two $k$-packets have intersection of 
size at most 1 . This operation $p_{K}$, when defined, is called a packet flip. When $p_{K}(\rho)$ is defined, we have

$$
\operatorname{Inv}\left(p_{K}(\rho)\right)= \begin{cases}\operatorname{Inv}(\rho) \backslash K & \text { if } K \in \operatorname{Inv}(\rho) \\ \operatorname{Inv}(\rho) \cup\{K\} & \text { otherwise. }\end{cases}
$$

We will now construct the set $B\left(I_{n}, k\right)$ as a quotient of $A\left(I_{n}, k\right)$ by a certain equivalence relation. Call $\rho, \rho^{\prime} \in A\left(I_{n}, k\right)$ elementarily equivalent if one can be obtained from the other by reversing the order of two neighboring elements that do not belong to a common $k$ packet. Let $\sim$ be the equivalence relation on $A\left(I_{n}, k\right)$ generated by these elementary equivalences, so that $\rho \sim \rho^{\prime}$ if and only if $\rho$ and $\rho^{\prime}$ can be connected by a sequence of elementary equivalences. Let

$$
B\left(I_{n}, k\right):=A\left(I_{n}, k\right) / \sim
$$

be the quotient of $A\left(I_{n}, k\right)$ by this equivalence relation. For $\rho \in A\left(I_{n}, k\right)$ let $[\rho]$ denote its class in $B\left(I_{n}, k\right)$. We set $r_{\min }=\left[\rho_{\min }\right]$ and $r_{\max }=\left[\rho_{\max }\right]$.

It is clear that if $\rho \sim \rho^{\prime}$ then $\operatorname{Inv}(\rho)=\operatorname{Inv}\left(\rho^{\prime}\right)$ and hence Inv descends to $B\left(I_{n}, k\right)$. We also extend the definition of $N$, defining $N(r)$ for $r \in B\left(I_{n}, k\right)$ by

$$
N(r)=\cup_{\rho \in r} N(\rho) .
$$

For $K \in N(r)$ there exists $\rho \in r$ with $K \in N(\rho)$, so that $p_{K}(\rho)$ is defined. It is clear then that $\left[p_{K}(\rho)\right]$ is independent of the choice of $\rho$, and we extend the definition of packet flips be defining $p_{K}(r)=\left[p_{K}(\rho)\right]$ for any $\rho \in r$ and $K \in N(\rho)$.

We now define the Manin-Schechtman higher Bruhat orders on the sets $B\left(I_{n}, k\right)$. For $r, r^{\prime} \in B\left(I_{n}, k\right)$, we write $r<_{M S} r^{\prime}$ if there exist sequences $K_{1}, \ldots, K_{m} \in C\left(I_{n}, k+1\right)$ and $r_{0}, \ldots, r_{m} \in B\left(I_{n}, k\right)$ such that $r=r_{0}, r^{\prime}=r_{m}, K_{i} \in N\left(r_{i-1}\right) \backslash \operatorname{Inv}\left(r_{i-1}\right)$, and $r_{i}=$ $p_{K_{i}}\left(r_{i-1}\right)$ for $1 \leqslant i \leqslant m$. The following theorem was proven by Manin and Schechtman about the relation $<_{M S}$ :

Theorem (cf. [4]). The following hold:

$-<_{M S}$ defines a partial order on $B\left(I_{n}, k\right)$.

- Under $<_{M S}, B\left(I_{n}, k\right)$ is a ranked poset with a unique minimal element, $r_{\min }$, and a unique maximal element, $r_{\max }$. The rank is given by $r \mapsto|\operatorname{Inv}(r)|$.

- The map $r_{\text {min }}<p_{K_{1}}\left(r_{\text {min }}\right)<\cdots<p_{K_{m}} \cdots p_{K_{1}}\left(r_{\text {min }}\right) \mapsto \rho: K_{1} \prec \cdots \prec K_{m}$ defines a bijection from the set of maximal chains in $B\left(I_{n}, k\right)$ to the set $A\left(I_{n}, k+1\right)$.

- The map Inv $: B\left(I_{n}, k\right) \rightarrow 2^{C\left(I_{n}, k+1\right)}$ is injective.

This is the central result that we wish to generalize to type $B$. First, we make explicit the connection of this construction for $k=1$ and $k=2$ with the type $A$ Weyl groups $S_{n}$. 


\section{Connection with Type A Weyl Groups}

As we saw before, for $k=1$ admissibility is a vacuous condition on orders of $I$, so $A\left(I_{n}, 1\right)$ is the set of total orderings of $I_{n}$. Furthermore any two distinct elements $i \neq j \in I_{n}$ belong to the common packet $\{i, j\}$ so there are no elementary equivalences between orderings, and $B\left(I_{n}, 1\right)=A\left(I_{n}, 1\right)$. We may then identify $B\left(I_{n}, 1\right)$ with the symmetric group $S_{n}$, where the total ordering $a_{1}<\cdots<a_{n}$ corresponds to the permutation $a_{i} \mapsto i$.

Let

$$
\Phi:=\left\{ \pm\left(e_{i}-e_{j}\right): 1 \leqslant i<j \leqslant n\right\} \subset \mathbb{R}^{n}
$$

be the root system attached to $S_{n}$, and choose the system of positive roots

$$
\Phi^{+}:=\left\{e_{i}-e_{j}: 1 \leqslant i<j \leqslant n\right\}
$$

with associated simple roots $\Pi=\left\{e_{i}-e_{i+1}: 1 \leqslant i<n\right\}$ and simple reflections $s_{i}=(i, i+1)$ for $1 \leqslant i<n$. There is then an obvious bijection between $C\left(I_{n}, 2\right)$ and $\Phi^{+}$given by

$$
\{i, j\} \mapsto e_{i}-e_{j}
$$

for $i<j$. We have a function Inv $: S_{n} \rightarrow 2^{\Phi^{+}}$given as usual by

$$
\operatorname{Inv}(w):=\left\{\alpha \in \Phi^{+}: w(\alpha) \notin \Phi^{+}\right\}
$$

so the length function $l: S_{n} \rightarrow \mathbb{Z}_{\geqslant 0}$ is $l(w)=|\operatorname{Inv}(w)|$. This is compatible with Inv : $B\left(I_{n}, 1\right) \rightarrow 2^{C\left(I_{n}, 2\right)}$ in the sense that the following diagram commutes:

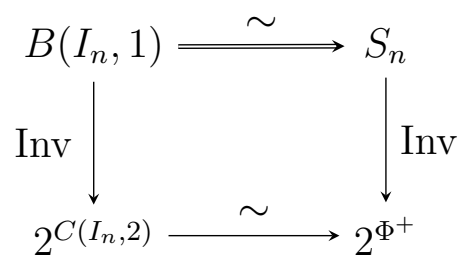

For an ordering $\rho=\left(a_{1}<\cdots<a_{n}\right)$ of $I_{n}$, corresponding to a permutation $w \in S_{n}$, the action of the packet flip $p_{\left\{a_{i}, a_{i+1}\right\}}$ for $i<n$ on $\rho$ corresponds to left multiplication by the adjacent transposition $s_{i}:=(i, i+1) \in S_{n}$. The ordering $<_{M S}$ on $B\left(I_{n}, 1\right)$ is thus identified with the weak left Bruhat order on $S_{n}$, defined by the covering relations $w<w^{\prime}$ for $l\left(w^{\prime}\right)=l(w)+1$ and $w^{\prime}=s_{i} w$ for some $i$.

The unique minimal element $r_{\min }$ of $B\left(I_{n}, 1\right)$ is identified with $1 \in S_{n}$ and the unique maximal element $r_{\max }$ is identified with the longest element $w_{0} \in S_{n}$ given by $w_{0}(i)=$ $n+1-i$. The identification of $A\left(I_{n}, 2\right)$ with the set of maximal chains in $B\left(I_{n}, 1\right)$ therefore identifies $A\left(I_{n}, 2\right)$ with the set of reduced expressions for $w_{0}$. Elementary equivalence in $A\left(I_{n}, 2\right)$ then corresponds to exchanging the positions of two adjacent commuting simple reflections $s_{i}$ and $s_{j}$ for $|i-j|>1$, and packet flip operations correspond to $m=3$ braid relations $s_{i} s_{i+1} s_{i}=s_{i+1} s_{i} s_{i+1}$. 
In the next section, we introduce a partial generalization of the Manin-Schechtman construction to type B. We provide analogues of the sets $C\left(I_{n}, k\right)$ and packets for all $k$ and $n$, and for $k=1,2$ and all $n$ we give a complete analogue, providing partially ordered sets analogous to the $B\left(I_{n}, k\right)$ satisfying the direct analogues of the main theorem of Manin and Schechtman on $<_{M S}$ and the root system combinatorics explained above in type $A$. In type $B, m=4$ (stst $=$ tsts) braid relations become relevant in addition to the $m=3$ $(s t s=t s t)$ braid relations seen in type $A$, leading to the introduction of two types of packets.

\section{Construction in Type B}

Let $E$ be a finite subset of $\mathbb{Z}$ stable under negation and not containing 0 . Let $E_{+}$denote the subset of positive elements in $E$. We give $E$ the total ordering inherited from $\mathbb{Z}$.

Definition 1. Let $C_{B}(E, 1):=E$. For $k>1$, let

$$
\begin{aligned}
& \widehat{C_{B}^{1}(E, k)}:=\{S \subset E:|S|=k,|S|=|\{|s|: s \in S\}|\}, \\
& C_{B}^{2}(E, k):=\left\{T \cup\{\star\}: T \subset E_{+},|T|=k-1\right\}
\end{aligned}
$$

(the condition in the first line means the elements of $S$ have distinct absolute values) where $\star$ is a bookkeeping symbol. $\mathbb{Z} / 2 \mathbb{Z}$ acts on $\widetilde{C_{B}^{1}(E, k)}$ by negation, and we let

$$
C_{B}^{1}(E, k)=\widetilde{C_{B}^{1}(E, k)} /(\mathbb{Z} / 2 \mathbb{Z}) .
$$

Finally, we set

$$
C_{B}(E, k)=C_{B}^{1}(E, k) \cup C_{B}^{2}(E, k) .
$$

Let $J_{n}=\{-n, \ldots,-1,1, \ldots, n\}$ for $n \geqslant 1$. The sets $C_{B}\left(J_{n}, k\right)$ are to play the roles of the sets $C\left(I_{n}, k\right)$ seen previously. We use the notation $C_{B}$ to indicate that these are constructions related to type $B$. As in the type $A$ case, we want to associate to an element $K \in C_{B}\left(J_{n}, k+1\right)$ a subset, $P_{B}(K) \subset C_{B}\left(J_{n}, k\right)$, called its packet. As before, a subset of $C_{B}\left(J_{n}, k\right)$ of the form $P_{B}(K)$ will be called a $k$-packet. $P_{B}(K)$ will be constructed differently depending on whether $K \in C_{B}^{1}\left(J_{n}, k\right)$ or $K \in C_{B}^{2}\left(J_{n}, k\right)$.

For brevity, we will define $\sigma$ to be the negation map $x \mapsto-x$.

Definition 2. For $K \in C_{B}^{1}\left(J_{n}, k+1\right)$, let $\left.R \in C_{B}^{1} \widetilde{\left(J_{n}, k\right.}+1\right)$ be a representative. Define $\widetilde{P}_{B}(K)$ to be the set of the $\sigma$-orbits of the $k$-element subsets of $R$. Clearly, $\widetilde{P}_{B}(K)$ is independent of the choice of representative $R$. Note that for $k=1$, the elements of $C_{B}(J, k)$ are not themselves $\sigma$-orbits. For this reason, we make the correction

$$
P_{B}(K):= \begin{cases}\cup_{T \in \widetilde{P}_{B}(K)} T & \text { if } K \in C_{B}^{1}\left(J_{n}, 2\right) \\ \widetilde{P}_{B}(K) & \text { if } K \in C_{B}^{1}\left(J_{n}, k+1\right) \text { for } k>1\end{cases}
$$


For example, if $K=[\{-3,2\}]$, then

$$
P_{B}(K)=\{-3,-2,2,3\} \subset C_{B}\left(J_{3}, 1\right),
$$

and if $K=[\{-3,2,1\}]$ then

$$
P_{B}(K)=\{[\{-3,2\}],[\{-3,1\}],[\{2,1\}]\} \subset C_{B}\left(J_{3}, 2\right) .
$$

For $K \in C_{B}^{2}\left(J_{n}, k+1\right)$, let $K^{\prime}=K \backslash\{\star\}$, and define $P_{B}(K):=C_{B}\left(K^{\prime} \cup \sigma\left(K^{\prime}\right), k\right)$.

Notation. As $C_{B}^{1}\left(J_{n}, k\right)$ is a set of equivalence classes, we encounter the problem of choosing a good way to represent its elements. We will denote the class of $\left\{a_{1}, \ldots, a_{k}\right\}$ by $\left[a_{1}, \ldots, a_{k}\right]$. Wherever possible, we will choose the representative for which the element with the greatest magnitude is negative. For some $T \in C_{B}^{1}\left(J_{n}, k\right)$, if $\left\{a_{1}, \ldots, a_{k}\right\}$ is such a representative, we will denote $T$ by the bracketed list $\left[a_{1}, \ldots, a_{k}\right]$. Such a representative will be referred to as a preferred representative, and we will indicate where this choice of representative is assumed.

For consistency, an element $s \in C_{B}^{2}\left(J_{n}, k\right)$ will also be denoted as a bracketed list $\left[b_{1}, \ldots b_{k-1}, \star\right]$, with either all $b_{i}$ positive or all $b_{i}$ negative.

We list elements with negative elements first, in increasing order, followed by positive elements, in decreasing order, e.g. $[-5,-2,3,1]$.

Definition 3. We now introduce certain standard orders on the sets $C_{B}\left(J_{n}, k\right)$ for $k=$ $1,2,3$.

The standard order of $C_{B}\left(J_{n}, 1\right)=J_{n}$ is the usual ordering of $J_{n}$ inherited from $\mathbb{Z}$.

The standard order of the set $C_{B}\left(J_{n}, 2\right)$ is defined with respect to preferred representatives. It is convenient here to represent elements $\left[a_{1}, \star\right]$ by $\left[-a_{1}, a_{1}\right]$. Viewing a total ordering as a list of elements read left to right, with the smallest elements occurring first, i.e. to the left, we define the standard order on $C_{B}\left(J_{n}, 2\right)$ as follows:

- Elements represented by two negative indices occur first, in lexicographic order.

- Elements with a single negative index occur afterwards. If the elements are listed in increasing order, $a_{1}, a_{2}$, then the ordering is lexicographic in the following sense:

$$
\left[a_{1}, a_{2}\right]<\left[b_{1}, b_{2}\right] \text { if } a_{1}<b_{1} \text {, or if } a_{1}=b_{1} \text { and } a_{2}>b_{2}
$$

Similarly, we have the following standard order for the set $C_{B}\left(J_{n}, 3\right)$. Similar to the above, it is convenient here to represent elements of the form $\left[a_{1}, a_{2}, \star\right]$ by $\left[-a_{1},-a_{2}, a_{2}\right]$ with $a_{1}>a_{2}>0$.

- Elements represented by three negative indices occur first, in lexicographic order.

- Elements represented by two negative indices occur second. For two elements represented by negative indices, define the order by setting $\left[a_{1}, a_{2}, a_{3}\right]<\left[b_{1}, b_{2}, b_{3}\right]$, where $a_{3}, b_{3}>0$, if and only if either $\left[a_{1}, a_{2}\right]<\left[b_{1}, b_{2}\right]$ or if both $\left[a_{1}, a_{2}\right]=\left[b_{1}, b_{2}\right]$ and $a_{3}>b_{3}$. 
- Elements represented by a single negative index occur third. For two elements of this form, we set $\left[a_{1}, a_{2}, a_{3}\right]<\left[b_{1}, b_{2}, b_{3}\right]$ if and only if either $a_{1}>b_{1}$ or if both $a_{1}=b_{1}$ and $\left[-a_{2},-a_{3}\right]<\left[-b_{2},-b_{3}\right]$.

The standard ordering of a given set is denoted $\rho_{\min }$ whenever this notation is unambiguous. For these sets, the reverse standard ordering, $\rho_{\max }$, is obtained by reversing the standard ordering.

Definition 4. For a $k$-packet $P$ with $k=2$ or $k=3$, the standard ordering of $P$ is given by the restriction of the standard ordering $\rho_{\min }$ to $P$. For a 1-packet, standard packet orderings are given by the following Hasse diagrams, where we assume $[j, i]$ is a preferred representative as above:

$\begin{array}{ccccc} & {[i, j]} & {[i,} & -j] & {[k, \star]} \\ i & -j & i & j & k \\ \uparrow & \uparrow & \uparrow & \uparrow & \uparrow \\ j & -i & -j & -i & -k\end{array}$

The reverse ordering of a 1-, 2-, or 3-packet $P$ is given by reversing the direction of each relation in the standard ordering, and Rev $P$ will denote the packet $P$ endowed with the reverse ordering.

Definition 5. A comparable component of a poset is defined to be a connected component of the poset's Hasse diagram.

Definition 6. For $k \leqslant 3$, we now make a collection of definitions analogous to the type A case:

- A total ordering $\rho$ of $C_{B}\left(J_{n}, k\right)$ is admissible if, for each $k$-packet $P, \rho$ extends either $P$ or Rev $P$.

- Let $A_{B}\left(J_{n}, k\right)$ be the set of all admissible orderings of $C_{B}\left(J_{n}, k\right)$. In particular $\rho_{\min }, \rho_{\max } \in A_{B}\left(J_{n}, k\right)$.

- For an admissible ordering $\rho$, let $\operatorname{Inv}(\rho)$ be the set of elements $K \in C_{B}\left(J_{n}, k+1\right)$ such that $\rho$ extends $\operatorname{Rev} P_{B}(K)$.

- For $\rho \in A_{B}\left(J_{n}, k\right)$, let $N(\rho)$ be the set of $K \in C_{B}\left(J_{n}, k+1\right)$ such that for each comparable component $C \subset P_{B}(K), C$ forms a chain in $\rho$.

- Two elements of $C_{B}\left(J_{n}, k\right)$ commute if they are incomparable in each $k$-packet to which they both belong.

- Two orderings $\rho, \rho^{\prime} \in A_{B}\left(J_{n}, k\right)$ are elementarily equivalent if $\rho^{\prime}$ can be obtained from $\rho$ by exchanging the order of two adjacent, commuting elements. 
- Let $\sim$ be the equivalence relation on $A_{B}\left(J_{n}, k\right)$ generated by elementary equivalence.

- Let $B_{B}\left(J_{n}, k\right)=A_{B}\left(J_{n}, k\right) / \sim$. Let $[\rho]$ denote the equivalence class of $\rho \in A_{B}\left(J_{n}, k\right)$.

- Let $N([\rho])=\cup_{\rho^{\prime} \in[\rho]} N\left(\rho^{\prime}\right)$.

As before, it is clear that $\rho \sim \rho^{\prime}$ implies $\operatorname{Inv}(\rho)=\operatorname{Inv}\left(\rho^{\prime}\right)$, so $\operatorname{Inv}$ descends to $B_{B}\left(J_{n}, k\right)$. Moreover, we have the following result, as in type A:

Proposition 7. The function Inv is injective on the set $B_{B}\left(J_{n}, k\right)$.

Proof. Consider two orderings $\rho$ and $\rho^{\prime}$, such that $\operatorname{Inv}(\rho)=\operatorname{Inv}\left(\rho^{\prime}\right)=S$. Note that the transitive closure of the union over the ordering relations $\operatorname{Rev} P$ for $P \in S$ and $Q$ for $Q \in C\left(J_{n}, k+1\right) \backslash S$ defines a poset structure on $C_{B}\left(J_{n}, k\right)$, and both $\rho$ and $\rho^{\prime}$ must be linear extensions of this poset. Furthermore, if two elements are incomparable in this poset, then they must be incomparable in every packet to which they both belong. But any two linear extensions of a finite poset differ by a sequence of transpositions of adjacent elements incomparable in the poset, so $\rho \sim \rho^{\prime}$ as needed.

Definition 8. Given $\rho$ in $A\left(J_{n}, k\right)$ and $K \in N(\rho)$, we can construct a new admissible order $p_{K}(\rho)$, the packet flip of $\rho$ by $K$, by reversing the order of each comparable component of $P_{B}(K)$ in $\rho$. Clearly

$$
\operatorname{Inv}\left(p_{K}(\rho)\right)= \begin{cases}\operatorname{Inv}(\rho) \backslash K & \text { if } K \in \operatorname{Inv}(\rho) \\ \operatorname{Inv}(\rho) \cup\{K\} & \text { otherwise. }\end{cases}
$$

Like in type A, we may extend this operation to $B_{B}\left(J_{n}, k\right)$ by acting on representatives. Specifically, for $r \in B_{B}\left(J_{n}, k\right)$ and $K \in N(r)$, there exists $\rho \in r$ with $K \in N(\rho)$, and we set $p_{K}(r)=\left[p_{K}(\rho)\right]$.

For any $[\rho],\left[\rho^{\prime}\right] \in B_{B}\left(J_{n}, k\right)$, we write $[\rho]<\left[\rho^{\prime}\right]$ if there exists a finite sequence $\left\{\rho_{i}\right\}_{1}^{m} \subset A_{B}\left(J_{n}, k\right)$ such that $\rho_{1}=\rho, \rho_{m}=\rho^{\prime}$, and for each pair $\left(\rho_{i}, \rho_{i+1}\right)$ there exists some $K_{i} \in N\left(\left[\rho_{i}\right]\right) \backslash \operatorname{Inv}\left(\left[\rho_{i}\right]\right)$ such that for some $\rho_{i}^{\prime} \in\left[\rho_{i}\right], \rho_{i+1}=p_{K_{i}}\left(\rho_{i}^{\prime}\right)$. This relation defines a partial ordering on the set $B_{B}\left(J_{n}, k\right)$.

Theorem 9. For the cases $k=1,2, B_{B}\left(J_{n}, k\right)$ has a unique maximal (respectively, minimal) element, given by $\left[\rho_{\max }\right]$ (resp. $\left.\left[\rho_{\min }\right]\right)$.

Our proof will make use of the following lemmas, which will be proved after the general argument is given.

Notation. For any admissible ordering $\rho \in A_{B}\left(J_{n}, k\right)$ and any subset $S \subset C_{B}\left(J_{n}, k\right)$, we write $\bar{S}(\rho)$ to denote the minimal chain containing $S$ in $\rho$. To simplify the notation, we write $S_{K}$ to denote the set $P_{B}(K)$.

Definition 10. For $\rho \in A_{B}\left(J_{n}, 2\right)$ we say that $x$ blocks $S$ in $\rho$ when $x \notin S$ but $x \in \bar{S}\left(\rho^{\prime}\right)$ for all $\rho^{\prime} \in[\rho]$. 
Lemma 11. Let a set $S \subset C_{B}\left(J_{n}, 2\right)$ be given, and let $\rho \in A_{B}\left(J_{n}, 2\right)$. If $x$ does not block $S$ in $\rho$ and $x \notin S$, then there exists some $\rho^{\prime} \in[\rho]$ such that $\bar{S}\left(\rho^{\prime}\right) \subset \bar{S}(\rho)$, and $x \notin \bar{S}\left(\rho^{\prime}\right)$.

Lemma 12. Let $\rho \in A_{B}\left(J_{n}, 2\right)$. For $K \in C_{B}\left(J_{n}, 3\right), K \notin N([\rho])$ if and only if there exists some $x$ which blocks $S_{K}$ in $\rho$.

Lemma 13. Let $[\rho] \in B_{B}\left(J_{n}, 2\right)$, and suppose $K \notin N([\rho]) \cup \operatorname{Inv}([\rho])$. Then at least one of the following seven cases holds for all $\rho^{\prime} \in[\rho]$ :

- If $K \in C_{B}^{1}\left(J_{n}, 3\right)$, let $[i, j, k]$ be a preferred representative. Then, we have either:

1. $[i, j]<[i, x]<[i, k]$,

2. $[i, k]<[k, x]<[j, k]$, or

3. $[i, j]<[j, x]<[j, k]$,

for some $x \in J_{n} \backslash\{i, j, k\}$.

- If $K \in C_{B}^{2}\left(J_{n}, 3\right)$, fix $K=[i, j, \star]$. Then, we have either:

1. $[i, j]<[i, x]<[i, \star]$,

2. $[i, \star]<[i, x]<[i,-j]$,

3. $[i,-j]<[j, x]<[j, \star]$, or

4. $[i, j]<[j, x]<[i,-j]$,

for some $x \in J_{n} \backslash\{i, j,-i,-j\}$.

Lemma 14. In the setting of the previous lemma, there exists some $K^{\prime} \in C_{B}\left(J_{n}, 3\right) \backslash$ Inv $([\rho])$ such that either $\overline{S_{K^{\prime}}}\left(\rho^{\prime}\right) \subsetneq \bar{S}_{K}\left(\rho^{\prime}\right)$ for all $\rho^{\prime} \in[\rho]$, or the minimal element of $S_{K^{\prime}}$ is greater than the minimal element in $S_{K}$ in every ordering $\rho^{\prime} \in[\rho]$.

Proof of Theorem 9. Clearly any class of orderings $[\rho]$ satisfying $\operatorname{Inv}([\rho])=C_{B}\left(J_{n}, k+1\right)$ must be maximal, so in particular $\left[\rho_{\max }\right]$ is a maximal element. By injectivity of Inv on $B_{B}\left(J_{n}, k\right),\left[\rho_{\max }\right]$ is the unique class of orderings for which $\operatorname{Inv}([\rho])=C_{B}\left(J_{n}, k+1\right)$. Therefore, we need only prove that any $[\rho] \in B_{B}\left(J_{n}, k\right)$ for which $\operatorname{Inv}([\rho]) \subsetneq C_{B}\left(J_{n}, k+1\right)$ is not maximal. Let $[\rho]$ be such an ordering, so we have some $K \in C_{B}\left(J_{n}, k+1\right) \backslash \operatorname{Inv}([\rho])$. We need to find $K^{\prime} \in N([\rho]) \backslash \operatorname{Inv}([\rho])$.

For $k=2$ this follows immediately from the preceding lemma and induction.

For $k=1$, the statement follows immediately from the identification in the next section (independently of the intervening material) of the poset $B_{B}\left(J_{n}, 1\right)$ and the Weyl group $B_{n}$ with the weak left Bruhat order. 
Proof of Lemma 11. Let $\rho, S$, and $x$ be as in the statement of the lemma. Then there exists $\hat{\rho} \in[\rho]$ such that $x \notin \bar{S}(\hat{\rho})$. By taking reverse orderings if necessary, we may assume $x<\min S$ in $\hat{\rho}$. Let $T$ be the subset of $\bar{S}(\rho)$ which is less than or equal to $x$ in the order $\rho$. Clearly $T$ forms a chain in $\rho$.

As $\hat{\rho} \sim \rho$, there exists a sequence $t_{1} \ldots t_{r}$ of pairs of commuting elements of $C_{B}\left(J_{n}, 2\right)$ such that $\hat{\rho}$ can be obtained from $\rho$ by exchanging the order of the pair $t_{1}$, then $t_{2}$, etc., where at each step the pair $t_{i}$ to be reversed is an adjacent pair. Let $t_{i_{1}}, \ldots, t_{i_{s}}$, with $1 \leqslant i_{1}<i_{2}<\cdots<i_{s} \leqslant r$ be the subsequence of pairs of elements in $T$. Then the ordering $\rho^{\prime}$ obtained from $\rho$ by first reversing the ordering of $t_{i_{1}}$, then $t_{i_{2}}$, etc., is such that $\bar{S}\left(\rho^{\prime}\right) \subset \bar{S}(\rho)$ and $x \notin \bar{S}\left(\rho^{\prime}\right)$.

Proof of Lemma 12. Fix $S=P_{B}(K)$. If some $x$ blocks $S$ in $\rho$, then certainly $K \notin N([\rho])$. Suppose that no element blocks $K$ in $\rho$. If $\bar{S}(\rho)=S$, then $K \in N([\rho])$. If not, there exists $y \in \bar{S}(\rho) \backslash S$. By Lemma 11, there exists $\rho^{\prime} \sim \rho$ such that $y \notin \bar{S}\left(\rho^{\prime}\right)$, and $\bar{S}\left(\rho^{\prime}\right) \subset \bar{S}(\rho)$, and the lemma follows by induction on $|\bar{S}(\rho)|$.

Proof of Lemma 13. Let $\rho \in A_{B}\left(J_{n}, 2\right)$ and $K \in C_{B}\left(J_{n}, 3\right) \backslash(N([\rho]) \cup \operatorname{Inv}(\rho))$. Write $K=[i, j, k]$, where $i<j<0$, by the conventions introduced earlier. Once again, let $S=P_{B}(K)$, and $\bar{S}(\rho)$ is the minimal chain containing $S$ in $\rho$.

First, we show that there exists some element which blocks $S$ in $\rho$, which does not commute with every element of $S$. Suppose to the contrary that every element which blocks $S$ commutes with every element of $S$. Then, by applying Lemma 11, we can produce some $\rho^{\prime} \in[\rho]$ for which the only elements in $\bar{S}\left(\rho^{\prime}\right) \backslash S$ are those which block $\rho$ in $S$. But then as these elements commute with every element of $S$ there exists an equivalent ordering $\rho^{*}$ for which $\bar{S}\left(\rho^{*}\right)=S$, contradicting $K \notin N([\rho])$.

Suppose $K \in C_{B}^{1}\left(J_{n}, 3\right)$. Then, we can conclude from the above that there exists some $b$ which blocks $S$ in $\rho$, and $b$ has the form $[i, x],[j, x]$, or $[k, x]$ for some $x \in J_{n} \backslash\{i, j, k\}$. Either $b$ falls into one of the stated cases, or one of the following:

1. $[i, j]<[k, x]<[i, k]$

2. $[i, k]<[i, x]<[j, k]$

In case $(1)$, consider the set $D=\{d:[i, j]<d<[k, x]\}$. If every element of $D$ commutes with $[i, j]$, then, as $b=[k, x]$ also commutes with $[i, j]$, there exists an equivalent ordering $\rho^{\prime} \sim \rho$ for which $\overline{S_{K}}\left(\rho^{\prime}\right)$ does not contain $b$, a contradiction. We may thus conclude that there is an element $b^{\prime} \in D$ of the form $[i, x]$ or $[j, x]$ such that $[i, j]<b^{\prime}<[i, k]<[j, k]$.

In case (2), consider the set $D=\{d:[i, k]<d<[j, k]\}$. If every element of $D$ commutes with $[j, k]$, then there exists an equivalent ordering $\rho^{\prime} \sim \rho$ for which $\overline{S_{K}}\left(\rho^{\prime}\right)$ does not contain $[i, x]$, a contradiction. We may thus conclude that there is an element $b^{\prime} \in D$ of the form $[k, x]$ or $[j, x]$ such that $[i, j]<[i, k]<b^{\prime}<[j, k]$.

If $K \in C_{B}^{2}\left(J_{n}, 3\right)$, then as above there exists some $b \in \bar{S}(\rho)$ of the form $[i, x]$ or $[j, x]$. Either $b$ belongs to one of the stated cases, or we have $[i,-j]<[i, x]<[j, \star]$. In this case, consider the set $D=\{d:[i,-j]<d<[j, \star]\}$. If every element of $D$ commutes with $[j, \star]$, then there exists an equivalent ordering $\rho^{\prime} \sim \rho$ for which $\overline{S_{K}}\left(\rho^{\prime}\right)$ does not 
contain $[i, x]$, a contradiction. We may thus conclude that there is an element $b^{\prime}$ of the form $[j, x]$ such that $[i,-j]<b^{\prime}<[j, \star]$.

Proof of Lemma 14. Lemma 14 is proved by case work. For the complete case analysis, refer to the Appendix [Sec. 6.1].

Theorem 15. For $k=1,2$, there is a bijection

$$
\left\{\text { maximal chains in } B_{B}\left(J_{n}, k\right)\right\} \stackrel{\sim}{\longrightarrow} A_{B}\left(J_{n}, k+1\right),
$$

defined by

$$
\left[\rho_{\text {min }}\right]=\left[\rho_{1}\right] \leqslant\left[\rho_{2}\right] \leqslant \cdots\left[\rho_{i}\right] \cdots \leqslant\left[\rho_{\max }\right] \mapsto K_{1}<\cdots<K_{m}
$$

where $\left[\rho_{i}\right] \in p_{K_{i-1}}\left(\ldots p_{K_{1}}\left(\left[\rho_{\text {min }}\right]\right)\right), m=\left|C\left(J_{n}, k+1\right)\right|$ and $K_{i} \in N\left(\left[\rho_{i}\right]\right) \backslash \operatorname{Inv}\left(\left[\rho_{i}\right]\right)$ for all $i$.

Proof. As $\left[\rho_{\min }\right]$ is the unique minimal element of $B_{B}\left(J_{n}, k\right)$ and $\left[\rho_{\max }\right]$ is the unique maximal element, the assignment

$$
\left[\rho_{\text {min }}\right]=\left[\rho_{1}\right] \leqslant\left[\rho_{2}\right] \leqslant \cdots\left[\rho_{i}\right] \cdots \leqslant\left[\rho_{\max }\right] \mapsto K_{1}<\cdots<K_{m}
$$

maps the set of maximal chains of $B_{B}\left(J_{n}, k\right)$ into total orderings of $C_{B}\left(J_{n}, 3\right)$, and this map is clearly injective. We need only show that its image is precisely $A_{B}\left(J_{n}, k+1\right)$. This reduces to checking a few cases, which is treated in the Appendix [Sec. 6.2].

\section{Connection with Type B Weyl Groups}

As before, let $J_{n}$ be the set $\{-n \ldots n\} \backslash\{0\}$. Recall that the Weyl group $B_{n}$ acts faithfully on $J_{n}$ as the set of permutations $\pi: J_{n} \rightarrow J_{n}$ such that $\pi(-i)=-\pi(i)$ for all $i \in J_{n}$. In this way, we have a natural inclusion $B_{n} \hookrightarrow S_{2 n}$. Likewise, the set $A_{B}\left(J_{n}, 1\right)=B_{B}\left(J_{n}, 1\right)$ of all admissible orderings of $J_{n}$ is contained in the set $A\left(J_{n}, 1\right)=B\left(J_{n}, 1\right)$ of all total orderings of $J_{n}$.

Definition 16. For a total ordering $\rho$ of $J_{n}$, say

$$
j_{-n}<j_{-n+1}<\cdots<j_{n-1}<j_{n},
$$

let $\pi_{\rho} \in S_{2 n}$ be the permutation of $J_{n}$ given by $j_{i} \mapsto i$. Let $\phi: B\left(J_{n}, 1\right) \rightarrow S_{2 n}$ denote the map $\rho \mapsto \pi_{\rho}$.

Proposition 17. The image of the composition $B_{B}\left(J_{n}, 1\right) \hookrightarrow B(J, 1) \stackrel{\phi}{\rightarrow} S_{2 n}$ is $B_{n}$

Proof. From the definitions, it is clear that the set of total orderings of $J_{n}$ sent into $B_{n}$ under $\phi$ are those reversed under negation. Certainly any such total ordering belongs to $B_{B}\left(J_{n}, 1\right)$, and we need only show the converse. So, let $\rho \in A_{B}\left(J_{n}, 1\right)$ be given, and let $x$ be the maximal element of $J_{n}$ with respect to $\rho$. Then, for every other element $y \in J_{n} \backslash\{x\}$, considering the packet order on $P_{B}([x, y])$, we have $y<x$ and hence $-x<-y$. So $-x$ is the minimal element, and the claim follows by induction on $n$. 
Recall the reflection representation of $B_{n}$ in $\mathbb{R}^{n}$ given by

$$
e_{i} \mapsto \operatorname{sign}(\pi(i)) \cdot e_{|\pi(i)|}
$$

for $\pi \in B_{n}$, where $e_{1}, \ldots, e_{n}$ is the standard basis of $\mathbb{R}^{n}$. We have the associated root system

$$
\Phi:=\left\{ \pm e_{i}: 1 \leqslant i \leqslant n\right\} \cup\left\{ \pm e_{i} \pm e_{j}: 1 \leqslant i<j \leqslant n\right\} \subset \mathbb{R}^{n} .
$$

We choose the set of positive roots

$$
\Phi^{+}:=\left\{e_{i}: 1 \leqslant i \leqslant n\right\} \cup\left\{e_{i} \pm e_{j}: i>j\right\}
$$

with associated set of simple roots

$$
\Pi:=\left\{e_{1}\right\} \cup\left\{e_{i}-e_{i-1}: 1<i \leqslant n\right\} .
$$

Under the realization of $B_{n}$ with the subgroup of the permutations of $J_{n}$ discussed above, the simple reflection $s_{e_{1}}$ is given by the permutation $(-1,1)$, and the simple reflection $s_{e_{i}-e_{i-1}}$ for $1<i \leqslant n$ is given by $(-i,-i+1)(i, i-1)$. We define the function

$$
\operatorname{Inv}: B_{n} \rightarrow 2^{\Phi^{+}}
$$

by

$$
\operatorname{Inv}(w)=\left\{\alpha \in \Phi^{+}: w(\alpha) \notin \Phi^{+}\right\}
$$

so that the length function $l: B_{n} \rightarrow \mathbb{Z}$ defined by the simple reflections $s_{\alpha}$ for $\alpha \in \Pi$ is given by $l(w)=|\operatorname{Inv}(w)|$.

We now give a specific bijection between $\Phi^{+}$and $C_{B}\left(J_{n}, 2\right)$ such that the definitions of Inv on $\Phi^{+}$and on $C_{B}\left(J_{n}, 2\right)$ become compatible with the identification of $B_{B}\left(J_{n}, 1\right)$ and $B_{n}$ :

Definition 18. Let $K \mapsto \alpha_{K}$ denote the bijection $C_{B}\left(J_{n}, 2\right) \rightarrow \Phi^{+}$given by

$$
\alpha_{K}= \begin{cases}e_{i}-e_{j} & \text { if } K=[i, j] \text { for } i>j>0, \\ e_{i}+e_{j} & \text { if } K=[i,-j] \text { for } i>j>0, \\ e_{k} & \text { if } K=[k, \star] \text { for } k>0\end{cases}
$$

Lemma 19. Let $\rho \in B_{B}\left(J_{n}, 1\right)$, and let $\pi_{\rho}=\phi(\rho) \in B_{n}$ be the corresponding element of $B_{n}$. Then for each $K \in C_{B}\left(J_{n}, 2\right)$, we have

$$
K \in \operatorname{Inv}(\rho) \Longleftrightarrow \pi_{\rho}\left(\alpha_{K}\right) \notin \Phi^{+} .
$$

In other words, the following diagram commutes.

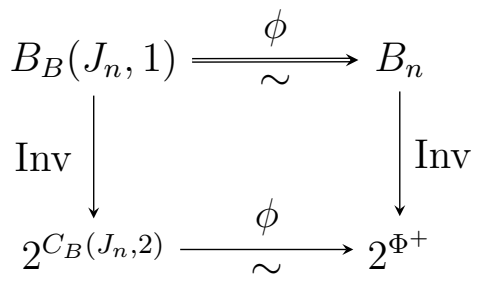


Proof. Let $\rho$ and $\pi_{\rho}$ be as in the statement of the lemma, and let $i, j \in\{1, \ldots, n\}$ with $i>j>0$. Let $k=\pi_{\rho}(i)$ and $l=\pi_{\rho}(j)$. Suppose that $[-j,-i] \notin \operatorname{Inv}(\rho)$, so that $k>l$. Then the image of the positive root $\alpha_{P}=e_{i}-e_{j}$ under $\pi_{\rho}$ is positive. In particular, either:

1. $k>l>0$, and $\pi_{\rho}\left(\alpha_{P}\right)=e_{k}-e_{l}$.

2. $k>0>l$, and $\pi_{\rho}\left(\alpha_{P}\right)=e_{k}+e_{-l}$, or

3. $0>k>l$, and $\pi_{\rho}\left(\alpha_{P}\right)=e_{-l}-e_{-k}$.

Conversely, if $[-i,-j] \in \operatorname{Inv}(\rho)$, so that $l>k$, we have one of the following:

1. $l>k>0$, and $\pi_{\rho}\left(\alpha_{P}\right)=e_{k}-e_{l}$.

2. $l>0>k$, and $\pi_{\rho}\left(\alpha_{P}\right)=-e_{-k}-e_{l}$, or

3. $0>l>k$, and $\pi_{\rho}\left(\alpha_{P}\right)=e_{-l}-e_{-k}$.

Therefore $\pi_{\rho}\left(\alpha_{P}\right)$ is not positive.

Next, we show that if $[-j, i] \notin \operatorname{Inv}(\rho)$, then the image of $\alpha_{P}=e_{i}+e_{j}$ under $\pi_{\rho}$ is positive. If this were the case, then we would have one of the following:

1. $-k<l<0$, and $\pi_{\rho}\left(\alpha_{P}\right)=e_{k}-e_{-l}$,

2. $-k<0<l$, and $\pi_{\rho}\left(\alpha_{P}\right)=e_{k}+e_{l}$, or

3. $0<-k<l$, and $\pi_{\rho}\left(\alpha_{P}\right)=e_{l}-e_{-k}$.

Analagous to the previous case, if $[-j, i] \in \operatorname{Inv}(\rho)$ then the image of $\alpha_{P}$ is not positive. The cases to consider here are:

1. $l<-k<0$, and $\pi_{\rho}\left(\alpha_{P}\right)=e_{k}-e-l$,

2. $l<0<-k$, and $\pi_{\rho}\left(\alpha_{P}\right)=-e_{k}-e_{l}$, or

3. $0<l<-k$, and $\pi_{\rho}\left(\alpha_{P}\right)=e_{l}-e_{-k}$.

Finally, it is clear that $\pi_{\rho}\left(e_{i}\right) \notin \Phi^{+}$if and only if $[i, \star] \in \operatorname{Inv}(\rho)$, as needed.

Recall that the weak left Bruhat order on $B_{n}$, with respect to the choice of positive roots $\Phi^{+}$, is the poset structure on $B_{n}$ with covering relations $w<w^{\prime}$ for $w^{\prime}=s w$ for some simple reflection $s$ with $l\left(w^{\prime}\right)=l(w)+1$.

Theorem 20. $\phi$ defines a poset isomorphism $B_{B}\left(J_{n}, 1\right) \rightarrow B_{n}$, where $B_{n}$ is ordered by the weak left Bruhat order.

Proof. Notice that under the bijection $\phi$, the action of packet flips on $B_{B}\left(J_{n}, 1\right)$ corresponds to left multiplications by simple reflections. The preceding lemma then shows that the covering relations in the two posets are identified under $\phi$, and the theorem follows. 
Corollary 21. $\phi$ induces a bijection $A_{B}\left(J_{n}, 2\right) \rightarrow R\left(w_{0}\right)$, where $w_{0}$ is the longest element of $B_{n}$ and $R\left(w_{0}\right)$ is the set of reduced expressions for $w_{0}$. Under this bijection, two admissible orderings $\rho, \rho^{\prime} \in A_{B}\left(J_{n}, 2\right)$ are elementarily equivalent if and only if the corresponding reduced expressions for $w_{0}$ are related by exchanging the order of a pair of adjacent commuting simple reflections. Packet flip operations $p_{K}$ on $A_{B}\left(J_{n}, 2\right)$ are identified with $m=3($ sts $=t s t)$ braid relations for $K \in C_{B}^{1}\left(J_{n}, 3\right)$ and are identified with $m=4($ stst $=$ tsts $)$ braid relations for $K \in C_{B}^{2}\left(J_{n}, 3\right)$.

\section{Appendix}

\subsection{Proof of Lemma 14}

As the lemma is merely casework, it was checked by a computer algorithm. We will describe this algorithm, and prove its correctness.

Definition 22. For some $\rho \in A_{B}\left(J_{n}, 2\right)$, and two elements $a, b \in C_{B}\left(J_{n}, 2\right)$, a crosses $b$ in $\rho$ if there exists $\rho^{\prime} \in[\rho]$ such that the relative positions of $a$ and $b$ in the orders $\rho$ and $\rho^{\prime}$ are opposite.

We first describe an algorithm which, on inputs $\rho \in A_{B}\left(J_{n}, 2\right)$ and $a, b \in C_{B}\left(J_{n}, 2\right)$, outputs 1 if $a$ crosses $b$ in $\rho$ and 0 otherwise.

Algorithm 1. If $b<a$ in $\rho$, replace $\rho$ by its reverse ordering. Let $S$ denote the chain of elements in $\rho$ greater than $a$ and less than $b$. Initialize a list, called right, containing only the element $a$. For each element $q$ in $S$, in ascending order, we compute whether $q$ commutes with every element in right. If so, we continue. If not, we add $q$ to right. Finally, return 1 if $b$ commutes with every $q$ contained in right, and 0 otherwise.

Proof of correctness. Suppose Algorithm 1 outputs 1. Then each element of $S \backslash\{a\}$ which is not added to right can be moved to the left past $a$, leaving only elements in right between $a$ and $b$. But $b$ commutes with all elements in right, so $b$ can be moved to the left past $a$, so $a$ crosses $b$ in $\rho$ as needed.

Conversely, suppose Algorithm 1 returns 0. Then there exists an element $q_{1}$ of right which does not commute with $b$. Either $q_{1}$ does not commute with $a$, or there exists $q_{2}$ in right with $q_{2}<q_{1}$ in the order $\rho$ such that $q_{1}$ and $q_{2}$ do not commute. Continuing in this manner, there is a sequence $q_{1}, \ldots, q_{s}$ for some $s \geqslant 1$ of elements of right such that $a<q_{s}<\cdots q_{1}<b$ in the order $\rho$ and each pair $\left(a, q_{s}\right),\left(q_{s}, q_{s-1}\right), \ldots,\left(q_{2}, q_{1}\right),\left(q_{1}, b\right)$ does not commute. It follows that the relative positions of these elements cannot change by transposing adjacent commuting elements, so in particular $a<b$ for all orders $\rho^{\prime} \in[\rho]$, so $a$ does not $\operatorname{cross} b$ in $\rho$.

In the following algorithm, posets are represented as directed acyclic graphs in which vertices represent elements of the poset and there is a directed edge for every covering relation. For elements $a, b, a<b$ exactly when there is a directed path from $a$ to $b$. The transitive union of two poset structures on the same set is given by the directed graph with the same vertex set and with edge set equal to the union of the edge sets for each 
poset. The resulting relation is reflexive and transitive, and it is antisymmetric so long as it contains no cycles. Linear extensions are computed using the topological sorting algorithm.

Notation. Recall that a 2-packet $P$ is understood to be an ordered set with the ordering inherited from the standard ordering $\rho_{\text {min }}$. Rev $P$ is understood to be the same set, with the ordering relation inherited from $\rho_{\max }$.

Each case from Lemma 13 involves a sequence $Q$ of 4 or 5 elements of $C_{B}\left(J_{n}, 2\right)$, all but one belonging to some packet $P_{B}(K)$ for $K \in C_{B}\left(J_{n}, 3\right)$, and the remaining element shares exactly one index with $K$. As such, there is a unique element $R \in C_{B}\left(J_{n}, 4\right)$ such that the set $T=\cup_{S \in P_{B}(R)} P_{B}(S)$ contains the sequence $Q$. Furthermore, the unique 2-packet containing any pair of elements in the sequence $Q$ is contained in $T$.

Algorithm 2. Initialize an empty list L. Each pair of elements pair appearing in the sequence $Q$ is contained in at most one common 2-packet $P$. For each such pair appearing in $Q$ and lying in the 2-packet $P$ :

- If pair appears in $Q$ in standard order, add the poset given by the standard order on $P$ to L.

- Otherwise, add the poset Rev $P$ given by the reverse-standard order to L.

Let $U$ be the set of packets $P_{B}(S)$ for $S \in P_{B}(R)$ whose order is not recorded in this manner. For each $B \subset U$, create a new list L' containing the elements of L. For each element $P \in U \cap B$, add the poset $P$ to L'. For each element $P \in U \backslash B$, add the poset Rev $P$ to L'. Compute the transitive union over the relations in L'. If there are no cycles, record a linear extension of the corresponding poset.

For each recorded linear extension, iterate over the packets $P_{B}(S)$ for $S \in P_{B}(R)$ until finding a 2-packet $P^{*}$ which is in standard order such that, with respect to the linear extension under consideration, either

- $\min _{\rho} P^{*}>\min _{\rho} P_{B}(K)$ and $\min _{\rho} P_{B}(K)$ does not $\operatorname{cross} \min _{\rho} P^{*}$, or

$-\min _{\rho} P^{*}=\min _{\rho} P_{B}(K), \max _{\rho} P^{*}<\max _{\rho} P_{B}(K)$, and $\max _{\rho} P^{*}$ does not cross $\max _{\rho} P_{B}(K)$.

If this is the case, the algorithm continues. Otherwise, it outputs 0 . If every linear extension recorded has been checked in this way, the algorithm outputs 1. Algorithm 2 returns 1 when run on each of the cases in Lemma 13, proving Lemma 14.

\subsection{Proof of Theorem 15}

We first show that the image of the map

$$
\left[\rho_{\min }\right]=\left[\rho_{1}\right] \leqslant\left[\rho_{2}\right] \leqslant \cdots\left[\rho_{i}\right] \cdots \leqslant\left[\rho_{\max }\right] \mapsto K_{1}<\cdots<K_{m}
$$

in question lies in $A_{B}\left(J_{n}, k+1\right)$. For this, we need to check that for every element $K \in C_{B}\left(J_{n}, k+2\right)$, its packet $P_{B}(K) \subset C_{B}\left(J_{n}, k+1\right)$ appears in either standard or 
reverse standard order in $K_{1}<\cdots<K_{m}$. For this, we look at the restriction of the standard order $\rho_{\text {min }}$ to the set $S=\cup_{Z \in P_{B}(K)} P(Z) \subset C_{B}\left(J_{n}, k\right)$ and consider the possible orders in which the packets of elements of $P_{B}(K)$ could be flipped. By inspection, we have the following tables indicating the possible orders in which the packets of elements of $P_{B}(K)$ can be flipped, which show in each case that the possible orders are exactly the standard or reverse standard order on $P_{B}(K)$. A preferred representative is assumed in the left-hand column only. For the case $K=[i, j, k]$ we consider only the subset $\{i, j, k\}$ of $S$, which is enough already to deduce the possible orderings of $P_{B}(K)$.

Case $\mathrm{k}=1$

\begin{tabular}{|l|l|l|}
\hline$K$ & Restriction of $\rho_{\min }$ to $S$ & Possible flip sequence (up to reverse) \\
\hline$[i, j, \star]$ & $-i<-j<j<i$ & {$[i, j] \prec[i, \star] \prec[i,-j] \prec[j, \star]$} \\
\hline $\begin{array}{l}{[i, j, k]} \\
\text { with } j<0\end{array}$ & $i<j<k$ & {$[i, j] \prec[i, k] \prec[j, k]$} \\
\hline $\begin{array}{l}{[i, j, k]} \\
\text { with } j>0\end{array}$ & $i<k<j$ & {$[j, k] \prec[i, j] \prec[i, k]$} \\
\hline
\end{tabular}

\section{Case $\mathrm{k}=2$}

\begin{tabular}{|c|c|c|}
\hline$K$ & Restriction of $\rho_{\min }$ to $S$ & Possible flip sequence (up to reverse) \\
\hline$[i, j, k, \star]$ & $\begin{array}{l}{[-i,-j]<[-i,-k]<} \\
{[-j,-k]<[i, \star]<[-i, j]<} \\
{[-i, k]<[j, \star]<[-j, k]<} \\
{[k, \star]}\end{array}$ & $\begin{array}{l}{[-i,-j,-k] \prec[i, j, \star] \prec[-i,-j, k] \prec} \\
{[-i,-k, j] \prec[i, k, \star] \prec[j, k, \star] \prec} \\
{[-i, j, k]}\end{array}$ \\
\hline $\begin{array}{l}{[i, j, k, l]} \\
\text { where } k<0\end{array}$ & $\begin{array}{l}{[i, j]<[i, k]<[j, k]<} \\
{[i, l]<[j, l]<[k, l]}\end{array}$ & {$[i j, k] \prec[i, j, l] \prec[i, k, l] \prec[j, k, l]$} \\
\hline $\begin{array}{l}{[i, j, l, k]} \\
\text { where } k>0\end{array}$ & $\begin{array}{l}{[i, j]<[-k,-l]<[i, l]<} \\
{[i, k]<[j, l]<[j, k]}\end{array}$ & {$[i, j, k] \prec[i, j, l] \prec[j, k, l] \prec[i, k, l]$} \\
\hline
\end{tabular}

In particular, we see that $K_{1} \cdots K_{m}$ is indeed an admissible ordering of $C_{B}\left(J_{n}, k+1\right)$.

Next we show surjectivity. Suppose $K_{N} \ldots K_{1}$ is an admissible order of $C_{B}\left(J_{n}, k+1\right)$. Let $r_{0}=\left[\rho_{\text {min }}\right]$ denote the class of the standard ordering of $C_{B}\left(J_{n}, k\right)$. We want to show that $K_{N} \ldots K_{1}$ gives a valid sequence of packet flips $p_{K_{N}} \ldots p_{K_{1}}$ on $r_{0}$. With the empty sequence of packet flips as base case, assume inductively that $p_{K_{i}} \ldots p_{K_{1}}$ is a valid sequence of packet flips on $r_{0}$ for some $i \geqslant 0$. Then writing $r_{i}=p_{K_{i}} \ldots p_{K_{1}}\left(r_{0}\right)$, we need to check that $K_{i+1} \in N\left(r_{i}\right)$. Noting that $K_{i+1}$ is the minimal element of $C_{B}\left(J_{n}, k+1\right) \backslash \operatorname{Inv}\left(r_{i}\right)$ with respect to the admissible order $K_{N} \ldots K_{1}$, it suffices to prove the following statement for $k=1,2$ : If $\rho$ is an admissible ordering of $C_{B}\left(J_{n}, k\right)$ and $K \in C_{B}\left(J_{n}, k+1\right) \backslash(\operatorname{Inv}(\rho) \cup$ $N([\rho]))$, then $K$ is not minimal in the restriction of any admissible ordering to $C_{B}\left(J_{n}, k+\right.$ 1) $\backslash \operatorname{Inv}(\rho)$. This is what we check by casework below. 
Case $\mathbf{k}=1$ Let $\prec$ denote the ordering of $J_{n}=C_{B}\left(J_{n}, 1\right)$ given by $\rho \in A_{B}\left(J_{n}, 1\right)$. Let $K=[k, l] \in C_{B}\left(J_{n}, 2\right) \backslash(\operatorname{Inv}(\rho) \cup N([\rho]))$ be as above, where if $K \in C_{B}^{1}\left(J_{n}, 2\right)$ then $[k, l]$ is a preferred representative, and if $K \in C_{B}^{2}\left(J_{n}, 2\right)$ then $l=-k>0$, by the convention used previously. As $[k, l] \notin \operatorname{Inv}(\rho) \cup N([\rho])$, there must exist $x \in J_{n}$ such that $k \prec x \prec l$. The following table considers the possible relative orderings of such $k, l$, and $x$ under the usual ordering of $\mathbb{Z}$, denoted $<$. The first column treats these relative positions of $x$, the second column lists implications about the order in which certain packets flips can be applied to $[\rho]$, and the final column lists the admissible order (up to reverse) of these packets, showing that each case leads to a contradiction, as needed.

\begin{tabular}{|l|l|l|}
\hline Condition & Implied order & Admissible order (up to reverse) \\
\hline$x<k<l$ & {$[x, k] \prec[k, l] \prec[x, l]$} & {$[x, k]<[x, l]<[k, l]$} \\
\hline$k<x<l$ & {$[k, l] \prec[k, x]$ and $[k, l] \prec$} & {$[k, x]<[k, l]<[x, l]$} \\
& {$[x, l]$} & \\
\hline$k<l<x$ & {$[l, x] \prec[k, l] \prec[k, x]$} & {$[k, l]<[k, x]<[x, l]$} \\
\hline
\end{tabular}

Case $\mathbf{k}=2$ Straightforward casework entirely analogous to the argument for $k=1$ given above proves the desired statement for $k=2$. In particular, let $\prec$ denote the ordering of $C_{B}\left(J_{n}, 2\right)$ given by some admissible ordering $\rho \in A_{B}\left(J_{n}, 2\right)$ and let $K \in$ $C_{B}\left(J_{n}, 3\right) \backslash(\operatorname{Inv}(\rho) \cup N([\rho]))$. We have either $K \in C_{B}^{1}\left(J_{n}, 3\right)$, in which case we can write $K=[k, l, m]$, or $K \in C_{B}^{2}\left(J_{n}, 3\right)$, in which case we can write $K=[i, j, \star]$. Then, by Lemma 13, there exists an $x \in J_{n}$ as specified in that lemma, leading to only 7 cases to consider. Each case may then be split into a few further cases, as we saw in the above analysis for $k=1$, according to the possible relative positions of $x$ among $k, l, m$ (in the case $K=[k, l, m]$ ) or $\pm i, \pm j$ (in the case $K=[i, j, \star]$ ) with respect to the usual ordering $<$ of the integers. In each of these several cases, one easily concludes that $K$ cannot be minimal in the restriction of any admissible ordering to $C_{B}\left(J_{n}, k+1\right) \backslash \operatorname{Inv}(\rho)$, as needed.

\section{Acknowledgements}

This paper represents the results of an undergraduate research project conducted by Suhas Vijaykumar in MIT's Summer Program in Undergraduate Research, with graduate student mentor Seth Shelley-Abrahamson. We thank Ben Elias for suggesting the idea of generalizing the Manin-Schechtman higher Bruhat orders to type B and for his guidance and excitement throughout the completion of this work, and we thank Daniel Thompson and Gabriella Studt for their initial work on this project. We also thank the anonymous referee for many useful comments, including some interesting suggestions for future work reflected in the introduction.

\section{References}

[1] B. Elias. Thick Soergel calculus in type A. Proceedings of the London Mathematical Society 112 (5): 924-978, 2016. 
[2] S. Felsner and H. Weil. A theorem on higher Bruhat orders. Discrete Comput Geom 23:121-127, 2000.

[3] M. Kapranov, V. Voevodsky. Free n-category generated by a cube, oriented matroids, and higher Bruhat orders. Funk. Analisys and Appl., 25:1, 50-52, 1990.

[4] Yu. I. Manin and V. V. Schechtman. Arrangements of hyperplanes, higher braid groups and higher Bruhat orders. Advanced Studies in Pure Mathematics, Iss. 17, pp. 298-308, 1989.

[5] V. Reiner and Y. Roichman. Diameter of graphs of reduced words and galleries. T. Am. Math. Soc., Vol. 365, No. 5, pp. 2779-2802, 2013.

[6] G. M. Ziegler. Higher bruhat orders and cyclic hyperplane arrangements. Topology, Vol. 42, No. 2, pp. 259-279, 1993. 